\title{
Evaluation of Proton Pump Inhibitors Prescribing among Non-Critically Ill Hospitalized Patients in a Malaysian Tertiary Hospital
}

\author{
Mohamed Hassan Elnaem*, Mohamad Haniki Nik Mohamed, Amirul Hazim bin Nazar, \\ Rabiatul Nur Khaliesa binti Ibrahim \\ Department of Pharmacy Practice, Faculty of Pharmacy, International Islamic University Malaysia, Kuantan, Pahang, Malaysia.
}

\section{ARTICLE INFO \\ Article history: \\ Received on: 27/05/2017 \\ Accepted on: 10/08/2017 \\ Available online: 30/12/2017}

\section{Key words:}

Proton pump inhibitors, Malaysia, prescribing patterns, Clinical practice guidelines, evaluation.

\begin{abstract}
Background: There are raised concerns regarding the rational guidelines-based prescribing of proton pump inhibitors (PPIs) therapy in the hospital setting.

Objective: To describe the current prescribing practice of PPIs among hospitalized patients in non-intensive care settings. Also, the study aimed to evaluate the appropriateness of the prescribed PPIs.

Methods: A cross-sectional study was conducted in the medical ward of a tertiary hospital in the state of Pahang, Malaysia. A total of 153 medical records of non-critically-ill adult patients receiving PPI therapy were investigated. The assessment of PPI prescribing was performed referring to clinical guidelines and the list of PPI-approved indications.
\end{abstract}

Results: The most frequently prescribed PPI regimen has been pantoprazole $40 \mathrm{mg}$ OD. Approximately $34 \%$ of the prescribed regimens were considered to be appropriate as it was consistent with the clinical guidelines. However, about $31 \%$ of the prescribed PPIs did not have a clear indication. Moreover, the inappropriate use of PPIs was revealed in nearly $19 \%$ of the total PPIs prescriptions. Finally, $16 \%$ of the prescribed PPIs were in need of dosage adjustment due to the presence of drug-drug interactions with the concurrently used medications.

Conclusion: Inappropriately prescribed PPIs are prevalent among non-critically ill-hospitalized adult patients in a Malaysian tertiary hospital. Initiatives for enforcing rational prescribing should focus on the proper documentation of evidence-based indications of PPIs in the medical records. There is a desperate need in the local setting for well-defined PPIs prescribing criteria. Future research should design and examine the impact of prescribing improvement interventions on the PPIs rational prescribing.

\section{INTRODUCTION}

As the most potent inhibitors of acid secretion, proton pump inhibitors (PPIs) are considered as the major treatment of acid-peptic disorders (Ali et al., 2009). Omeprazole is ranked on the top four among the highest expenditure of the forty most utilized drugs in the Malaysian clinical settings (Lim and Mohamed Izham, 2012). It has been reported that lack of appropriate guidelines had contributed to the rise in the relatively irrational PPIs prescribing in particular for prophylactic purposes

\footnotetext{
* Corresponding Author

Email: drmelnaem @ gmail.com
}

(Oh et al., 2015). Consequently, studies to evaluate PPIs prescription patterns among hospitalized patients were warranted (Barnes, 2015; Nardino et al., 2000; Niklasson et al., 2003). The assurance of the proper prescribing of PPIs is substantive to avoid the potential risk associated with long-term PPIs use for those who failed to get their regular follow-up examinations (Atkins et al., 2013). Potential adverse effects that may occur in patients treated with PPI for an extended period were hypergastrinemia, diarrhea, and pneumonia due to bacterial infection, and impaired nutrient absorption (Ali et al., 2009; Atkins et al., 2013; Tleyjeh et al., 2012). The appropriate use of PPIs, especially among hospitalized patients, remains a major issue among the health care professionals 
touching on the indication and duration of the therapy (Ntaios et al., 2009). In a US study among hospitalized patients, it was found that $59 \%$ of the admitted patients received acid-suppressant medications although the risk of nosocomial gastrointestinal bleeding was relatively rare outside intensive care settings. The research findings did not support the routine prophylactic use of PPIs among non-critically ill patients (Herzig et al., 2011). Moreover, previous studies had reported inappropriate prescription of PPIs to patients who did not have a valid indication to receive such therapy (Haroon et al., 2013; Leri et al., 2013). In a study conducted in Spain, it has been shown that out of the 328 patients, the rate of PPIs prescriptions without proper indications were $74.47 \%$ of admitted patients, $61.25 \%$ in those who have to stay in the hospital and $80.24 \%$ after they had been discharged (Ramirez et al., 2010). Also, the results of one US study showed that half of the all hospitalized patients had been prescribed with acidsuppressive medication although no valid indication supported PPIs prescribing (Nardino et al., 2000). Furthermore, it was demonstrated that the prescribing pattern of the acid-suppressive drug to treat minor symptoms without a proper indication, is also common (Bashford et al., 1998). Stress ulcer prophylaxis (SUP) is one of the most common indications for PPI among critically ill patients. In some settings, SUP indication was extended unnecessarily to justify the PPIs use among non-critically ill patients despite the presence of clear guidelines needed to be abided by the health care professionals in determining the eligibility of patients for this indication (Arthur et al, 2007). According to a study done by Farley et al. the patient had been unnecessarily given PPIs as SUP in the ICU and continued receiving PPIs beyond the ICU setting including ward patients and discharged patients without any suitable indication (Farley et al., 2013). Also, in a Jordanian study that aimed to assess the incidence of PPIs overprescribing for SUP; it has been found that the majority of the patients were irrationally given PPI (Alqudah et al., 2016). The overutilization of PPIs therapy may increase the incidence of potential adverse drug reactions and raise the cost of treatment for both hospital management and patient (Farrell et al., 2010). PPIs overuse may potentially lead to bone fractures, headaches, and gastrointestinal infections (Ramirez et al., 2010). According to one US study conducted in a university hospital, the inappropriate PPIs use contributed to potential increased cost of USD 37000 per year without involving the cost associated with potential risks of unnecessary therapy (Belfield et al., 2017). The cost burden associated with PPIs misuse can be minimized with the pharmacist intervention in reviewing prescribing orders for proper indications, route of administration and appropriate timing for switching from oral to intravenous (IV) preparations (Nasser et al., 2010). Moreover, it was highlighted that the physicians should advise their patients to elude the unnecessary use of PPIs because of its safety concerns particularly in the patients who are more prone to osteoporotic fracture (Yu et al., 2011). This work was directed to provide updates regarding the current practice of PPIs prescribing among non-critically-ill hospitalized patients in a Malaysian health care facility. Also, we aimed to evaluate the appropriateness of PPIs prescribing among hospitalized patients. The research team proposed to use these study findings to create awareness among healthcare professionals about rational prescribing of PPIs.

\section{MATERIALS AND METHODS}

\section{Study Design and Setting}

A cross-sectional study was conducted in the main medical ward of a Malaysian tertiary hospital between October to December 2016. The research team investigated the medical records of hospitalized non-critically-ill patients who were receiving PPI therapy.

\section{Ethical consideration}

The study protocol obtained the ethical approval from the Malaysian National Medical Research Register NMRR-16-1781332849 (IIR). All the rules and regulations to protect patient privacy had been strictly followed during the research.

\section{Study Sample}

A total of 153 medical records of hospitalized patients were investigated focusing on the prescribing of a PPI. Data collection was done using convenience sampling. Inclusion criteria were the adult hospitalized patients prescribed with proton pump inhibitors during their admission in non-critical settings. The exclusion criteria were pediatric patients and adult critically ill patients prescribed with proton pump inhibitor.

\section{Study Instrument}

The study instrument is a self-designed data collection form. The documentation involved patients' demographic data (age, gender, race, pregnancy, lactation, and concomitant disease) and drug usage (drug name, dose, dosage form, frequency, duration, treatment indications and concurrent medications). In light of the absence of clear institutional guidelines, the National Institute for Health, and Care Excellence (NICE) guidelines and the US Food and Drug Administration (FDA) list of PPI-approved indications were considered as our references in the assessment of PPIs prescriptions. The evaluation of PPI prescribing was classified into four principal categories, which were "Appropriate," "Inappropriate", "Need adjustment" and "Unclear." PPI prescription was classified as appropriate when it was done according to the selected guidelines and it was classified as inappropriate if it was not done in compliance with the guidelines. The third category involved those prescriptions with drug-drug interactions between PPI and other medications that may require dose adjustment. Finally, the unclear classification was applied to PPIs prescriptions that lack clear justification or indication.

\section{Statistical Analysis}

The data were analyzed using SPSS software version 23. Descriptive statistics (frequencies and percentages) were used to present the findings. 


\section{RESULTS}

The total number of patients' records investigated was 160. Seven data collection forms were excluded because of not fulfilling the inclusion criteria. Data from 153 records were included for analysis. An overview of demographics of the study sample regarding their gender, race, and age are shown in Table $\mathbf{1 .}$

Table 1: Demographic data of the study participants.

\begin{tabular}{|c|c|c|}
\hline Characteristic & Frequency (N) & Percentage (\%) \\
\hline \multicolumn{3}{|c|}{ Gender } \\
\hline Male & 80 & $52.3 \%$ \\
\hline Female & 73 & $47.7 \%$ \\
\hline \multicolumn{3}{|c|}{ Race } \\
\hline Malay & 118 & $77.1 \%$ \\
\hline Chinese & 25 & $16.3 \%$ \\
\hline Indian & 10 & $6.5 \%$ \\
\hline \multicolumn{3}{|c|}{ Age } \\
\hline $18-20$ & 11 & $7.2 \%$ \\
\hline $20-44$ & 23 & $15 \%$ \\
\hline $45-65$ & 82 & $53.6 \%$ \\
\hline Older than 65 & 37 & $24.2 \%$ \\
\hline Total & 153 & 100 \\
\hline
\end{tabular}

The most frequently prescribed PPI regimen was pantoprazole 40mg OD $(n=62,40.5 \%)$. Whereas, the least prescribed PPIs regimens were pantoprazole 20mg OD, omeprazole $20 \mathrm{mg}$ bid, esomeprazole $40 \mathrm{mg}$ bid and esomeprazole $40 \mathrm{mg}$ OD. Only one prescription was reported for each of these regimens. The frequency distribution of the PPI regimens among hospitalized non-critically ill patients in our setting are displayed in Table 2.

Table 2: PPI Drug regimens.

\begin{tabular}{ccc}
\hline PPI Regimen & Frequency (N) & Percentage (\%) \\
\hline Pantoprazole 40mg OD & 62 & 40.5 \\
Omeprazole 20mg OD & 33 & 21.6 \\
Omeprazole 40mg OD & 32 & 20.9 \\
Pantoprazole 20mg OD & 1 & .7 \\
Pantoprazole 40mg BD & 22 & 14.4 \\
Omeprazole 20mg BD & 1 & .7 \\
Esomeprazole 40mg BD & 1 & .7 \\
Esomeprazole 40mg OD & 1 & .7 \\
\hline Total & $\mathbf{1 5 3}$ & $\mathbf{1 0 0}$ \\
\hline
\end{tabular}

Regarding the frequency of comorbidities among our study cases, hypertension marked the highest frequency followed by diabetes mellitus. COPD had the lowest frequency among other concomitant diseases. An overview of concomitant diseases in patients receiving PPI therapy with their reported percentages is shown in Figure 1.

PPIs were mostly being prescribed for gastritis, concurrent use with other medicines (e.g., NSAIDS), followed by gastrointestinal bleeding and GERD. Meanwhile, the esophagealrelated diseases such as esophageal varices, Barret's esophagus, and erosive esophagitis, as well as acute pancreatitis were the least indications that required PPIs prescription. A list of PPIs indications is shown in Figure 2.

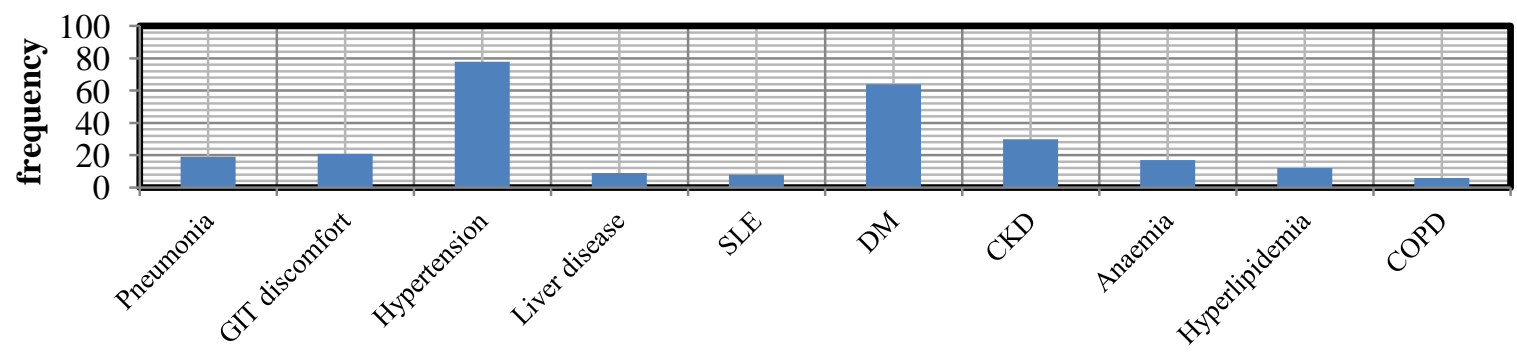

Fig. 1: Concomitant diseases in patients receiving PPIs. *Abbreviation: $\mathrm{SLE}=$ Systemic Lupus Erythromatosus, $\mathrm{CKD}=$ Chronic Kidney Disease $\mathrm{DM}=$ Diabetes Mellitus, $\mathrm{COPD}=$ Chronic Obstructive Pulmonary Disease

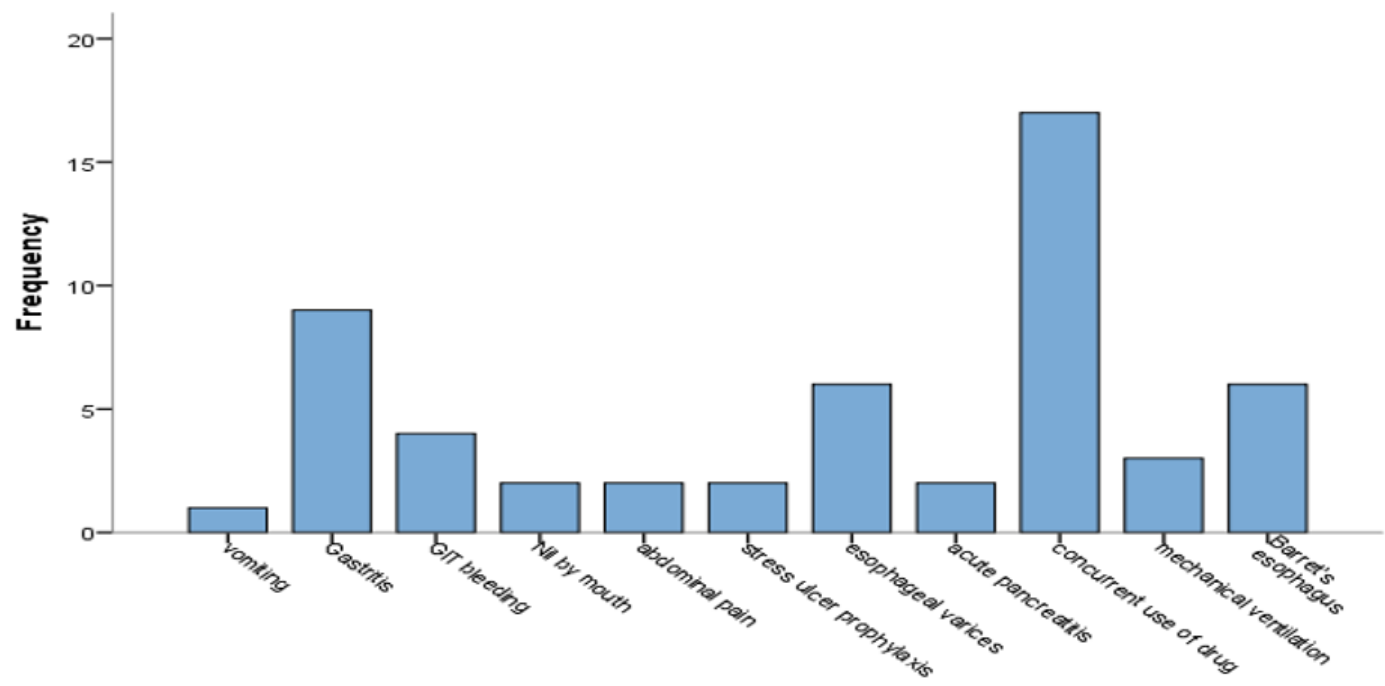

Fig. 2: Indications for PPIs prescribing. 


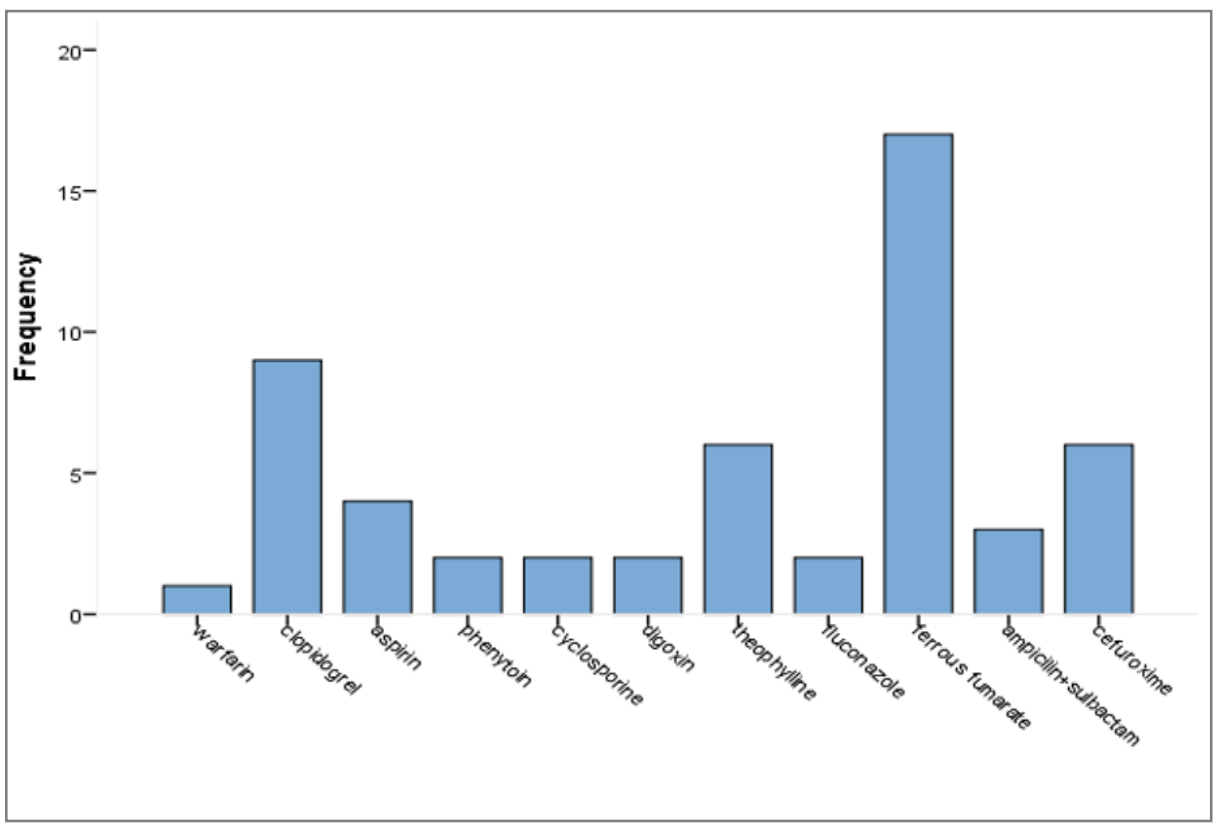

Fig. 3: Drugs that potentially interact with the prescribed PPIs.

Following the screening for the potential drug interactions, $35.3 \%$ of the cases $(\mathrm{N}=54)$ who were prescribed with PPI during their hospitalization period had also taken medications that may interact with their PPI regimen. The findings underscored 11 possible drugs that can interact with PPIs. Ferrous fumarate had the highest frequency compared to other drugs (17 out of 54 patients). Warfarin marked the lowest frequency that was only one patient had taken it out of 54 patients. A complete list of potentially interacting drugs is illustrated in Figure 3.

Not all the concurrently used medications had the same degree of interacting with PPIs. Severe drug-drug interactions were identified in seven medications. Warfarin, the least common co-prescribed medicine, interacted moderately with PPIs. Three medications had only minor interactions with PPIs. Further classification of co-prescribed medicines according to their interaction degree with PPIs is presented in Table 3.

Table 3: Classification of degree of drug interactions with PPI

\begin{tabular}{lll}
\hline $\begin{array}{c}\text { Mild } \\
(\mathbf{1 3} ; \mathbf{8 . 5 \%})\end{array}$ & $\begin{array}{c}\text { Moderate } \\
(\mathbf{1 ;} \mathbf{0 . 7 \%})\end{array}$ & \multicolumn{1}{c}{ Severe (40; 26.1\%) } \\
\hline Theophylline & Warfarin & Clopidogrel \\
Fluconazole & & Ampicillin + Sulbactam (Unasyn) \\
Aspirin & & Phenytoin \\
& & Ferrous fumarate \\
& & Cefuroxime \\
& & Cyclosporine \\
& & Digoxin \\
\hline
\end{tabular}

Approximately $34 \%$ of the prescribed regimens were considered to be appropriate as they complied with the clinical guidelines. Whereas, about $31 \%$ of the prescriptions did not have a clear indication. Moreover, the inappropriate use of PPI was revealed in nearly $19 \%$ of the total prescriptions. Finally, a total of $15.7 \%$ of the prescribed PPIs were in need of dosage adjustment due to the presence of drug-drug interactions between
PPI and the concurrently used medications. An outline of the outcomes of the evaluation of the prescribed PPI regimens is shown in Table 4.

Table 4: Evaluation of the prescribed PPI Regimens.

\begin{tabular}{ccc}
\hline Evaluation of PPI Regimen & $\begin{array}{c}\text { Frequenc } \\
\mathbf{y}(\mathbf{N})\end{array}$ & $\begin{array}{c}\text { Percentage } \\
(\boldsymbol{\%})\end{array}$ \\
\hline Appropriate-according to guideline & 52 & 34.0 \\
Inappropriate-not stated in guideline & 29 & 19.0 \\
Need dose adjustment due to DDI* & 24 & 15.7 \\
Unclear- no clear indication stated & 48 & 31.4 \\
Total & $\mathbf{1 5 3}$ & $\mathbf{1 0 0}$ \\
\hline
\end{tabular}

*DDI: drug-drug interaction.

\section{DISCUSSION}

\section{Indications of PPIs}

To our knowledge, this is the first attempt to highlight the assessment of PPIs prescribing in the state of Pahang, Malaysia. The three commonly used proton pump inhibitors in our settings are pantoprazole, omeprazole, and esomeprazole. The regimen for each PPI is described in details for each indication. Most PPIs are available in at least two dosage regimens.

The US-FDA approved PPIs indications include erosive esophagitis, gastroesophageal reflux disease (GERD), gastric ulcer associated with nonsteroidal anti-inflammatory drugs (NSAIDs), dyspepsia, H. pylori eradication and Zollinger-Ellison (ZE) syndrome. Evaluation of the prescribing appropriateness was based on this list of PPIs approved indications. All these indications are supported by Greek National Drug Organization and have been used in the study done by Ntaios and his colleagues (Ntaios et al., 2009). Our study showed that only 19\% of PPIs prescribed to our patients had no relevant indications based on USFDA-approved criteria. Meanwhile, the rest of the cases had PPIs prescribed based on justified indication (34\%) or unclear reasons $(31 \%)$. In a study conducted in the US regarding appropriate use of 
PPIs, it was found that $30 \%$ of the study cases were given PPIs for no documented indications (George et al., 2008).

From this study, it is evident that PPIs were mostly indicated for gastritis, followed by vomiting, nil by mouth and GIT bleeding with the percentage of $10.5 \%, 7.2 \%, 6.5 \%$, and $5.9 \%$ respectively. The clinical benefits attained by the use of PPI in patients with gastritis have been highlighted in the literature (Suzuki et al., 2008). Similarly, in a systematic review done by Pinto et al., they underpinned that PPIs were regarded as the first line treatment in $H$. pylori positive patients who were diagnosed with functional dyspepsia (Pinto-Sanchez et al., 2014) A closer inspection into the frequency of PPIs prescriptions on the real indications reveals that PPIs were also indicated for patients presented with GIT bleeding. This indication is a common one in our study settings. Furthermore, it can be related to a study that underscored the administration of intravenous PPI as an effective strategy to prevent the recurrence of GIT bleeding in most patients (Cheng and Sheu, 2011).

\section{Concomitant Disease}

\section{Hepatitis}

Several case reports in the literature highlighted the issue of liver abnormalities in patients taking PPI regimen. In one case report, it was stated that patient developed hepatitis after four weeks of consuming omeprazole; manifested as the transient elevation of the hepatic enzymes that went back to normal after stop taking omeprazole (Koury et al., 1998). A similar case was also reported with the use of pantoprazole, which induced severe acute hepatitis in a patient who consumed PPI for one month with her corticosteroid therapy. After discontinuing the pantoprazole treatment for one week, her liver function improved (Sandig et al., 2011). Liver disease contributes to about $5.9 \%$ from all the concomitant disease that occurred concurrently with PPI prescription. In line with the previously reported cases, the findings highlight the potential association between PPI use and the incidence of acute hepatitis that may need further investigation.

\section{Systemic Lupus Erythematous (SLE)}

Prescriptions of PPIs were common among the majority of patients who were diagnosed with systemic lupus erythematosus (SLE). This finding could be linked to what has been stated in the literature regarding the reasons behind the use of PPIs in a patient with SLE. A common cause was the frequent incidence of anorexia, nausea, and vomiting which have been seen in $50 \%$ of the lupus patients ( $\mathrm{Xu}$ et al., 2010). According to previous research, gastritis is commonly observed in patients with SLE is one of the common indications for PPI therapy (Musaev et al., 1991). Moreover, gastrointestinal discomfort may occur due to the effect of medications used for the disease management. For example, the high-dose steroid may mask the symptoms of peptic ulcer perforation which have been reported among patients with SLE (Medina et al., 1997).

\section{Appropriate Use of PPI}

The appropriateness of the use of PPIs was based on several guidelines including NICE pathway guidelines and the USFDA-list of PPI-approved indications. Depending on the results shown, the percentage of appropriate use of PPIs was $34 \%$ of the total prescriptions. This result is almost similar to an Australian study that reported the proper use of PPIs in about $37 \%$ of the study cases according to the Australian Schedule of Pharmaceutical Benefits criteria (Naunton et al., 2000). Additionally, the result of an Irish study reported a percentage of $30 \%$ of total PPI prescriptions complied with British National Formulary (BNF) guidelines (Molloy et al., 2010). However, in a Singaporean study with a larger sample size than our study, it has been found that a total of $45.9 \%$ of the study participants were prescribed with PPIs appropriately according to the FDA-approved indication (Tze et al., 2014). It is noticeable that different studies have used different assessment criteria for the proper PPI use. The reported percentages of appropriate PPI use were not exceeding $50 \%$ of the overall number of cases in all reported findings, which indicate the need for more effort towards optimizing the use of PPIs in the hospital setting.

\section{Inappropriate Use of PPI}

It is reported in our findings that $19 \%$ of the inpatients were prescribed with PPIs inappropriately. The findings contradict a few previous studies that revealed a higher percentage of the inappropriate prescriptions of PPIs among hospitalized patients (Leri et al., 2013; Molloy et al., 2010). In our settings, PPIs were frequently given to patients who had anemia. Based on the guidelines, anemic patients are not recommended to be routinely prescribed with PPI because it may result in gastric acid hyposecretion that may affect the iron absorption (Ali et al., 2009). Thus, PPIs prescribing to anemic patients could worsen the condition and deemed as inappropriate.

\section{Unclear Usage of PPI}

The major issue discovered by this study is the poor documentation of PPIs prescribing among the inpatients. In our research, there is nearly $30 \%$ of all PPIs prescription were found to have no clear indication. An Irish study aimed to evaluate PPIs use among medical inpatients has reported a similar problem of poor documentation, but with a higher percentage of $45 \%$ (Haroon et al., 2013). In another study, the lack of proper documentation of PPIs uses was found in nearly $40 \%$ of the study cases where no clear indication for the PPI use was documented in the patients' records (Tze et al., 2014). It is evident that when the indications for prescribing PPIs are not justified clearly in the inpatients' records, the assessment process will be less precise and the decision of appropriateness will be difficult to be determined. Consequently, the insufficient documentation may lead to underestimation of the number of eligible patients to receive PPI therapy according to the list of approved indications. 


\section{Limitation of the study}

This study has several limitations. The first limitation was the time constraint. This study was executed over only a twomonth period. The relatively small sample size may be the second limitation of our work although it is comparable to similar studies in the literature. The study was involving only one tertiary hospital in the state of Pahang, Malaysia, so, the findings could not represent other clinical settings in the country.

\section{CONCLUSION}

There are opportunities for the potential enhancement of PPIs prescribing practice. Proper screening of co-administered medications may help to decrease the likelihood of drug-drug interactions. Initiatives for enforcing rational prescribing should focus on the proper documentation of evidence-based indications of PPIs in the medical records. There is a desperate need in the local setting for well-defined PPIs prescribing criteria. Future research will be required to delineate the determinants of inappropriate PPI prescribing in the Malaysian clinical settings and examine the impact of prescribing improvement interventions on the PPIs rational prescribing.

\section{Financial support: Nil.}

Conflict of Interests: There are no conflicts of interest.

\section{REFERENCES}

Ali T, Roberts DN, Tierney WM. Long-term Safety Concerns with Proton Pump Inhibitors. Am J Med. 2009;122:896-903.

Alqudah MAY, Al-azzam S, Alzoubi K, Alkhatatbeh M, Rawashdeh N. Overuse of proton pump inhibitors for stress ulcer prophylaxis in Jordan. Int J Clin Pharmacol Ther. 2016:1-6.

Arthur Grube RR, May DB. Stress ulcer prophylaxis in hospitalized patients not in intensive care units. Am J Heal Pharm. 2007; 64:1396-400.

Atkins AM, Sekar MC, Ph R. Proton Pump Inhibitors: Their Misuse, Overuse, And Abuse. IOSR J Pharm. 2013;3:25-9.

Barnes MN. Overuse of Proton Pump Inhibitors in the Hospitalized Patient. US Pharm. 2015; 40:12-25.

Bashford JN, Norwood J, Chapman SR. Why are patients prescribed proton pump inhibitors? Retrospective analysis of link between morbidity and prescribing in the General Practice Research Database. Bmj. 1998; 317:452-6.

Belfield KD, Kuyumjian AG, Teran R, Amadi M, Blatt M, Bicking K. Impact of A Collaborative Strategy to Reduce the Inappropriate Use of Acid Suppressive Therapy in Non-Intensive Care Unit Patients. Ann Pharmacother. 2017; 51:577-83.

Cheng H-C, Sheu B-S. Intravenous proton pump inhibitors for peptic ulcer bleeding: Clinical benefits and limits. World J Gastrointest Endosc. 2011; 3:49-56.

Farley KJ, Barned KL, Crozier TM. Inappropriate continuation of stress ulcer prophylaxis beyond the intensive care setting. Crit Care Resusc. 2013;15:147-51.

Farrell CP, Mercogliano G, Kuntz CL. Overuse of stress ulcer prophylaxis in the critical care setting and beyond. J Crit Care [Internet]. 2010; 25:214-20.
George CJ, Rph MO, Kore B, Ph MO, Ross JS, Mhs MO. Appropriate Proton Pump Inhibitor Use Among Older Adults: A Retrospective Chart Review. Am J Geriatr Pharmacother. 2008; 6:249-54.

Haroon M, Yasin F, Gardezi SKM, Adeeb F, Walker F. Inappropriate use of proton pump inhibitors among medical inpatients: a questionnaire-based observational study. JRSM Short Rep. 2013; 4:36.

Herzig SJ, Vaughn BP, Howell MD, Ngo LH, Marcantonio ER. Acid-suppressive medication use and the risk for nosocomial gastrointestinal tract bleeding. Arch Intern Med. 2011; 171:991-7.

Koury SI, Stone CK, La Charité DD. Omeprazole and the development of acute hepatitis. Eur J Emerg Med. 1998; 5:467-9.

Leri F, Ayzenberg M, Voyce SJ, Klein A, Hartz L, Smego RA, et al. Four-Year Trends of Inappropriate Proton Pump Inhibitor Use After Hospital Discharge. South Med J. 2013; 106:270-3.

Lim L, Mohamed Izham MI. Selection of proton pump inhibitors (PPIS) for formulary inclusion using an objective scoring system in Malaysia. J Appl Pharm Sci. 2012;2:17-24.

Medina F, Ayala A, Jara LJ, Becerra M, Miranda JM, Fraga A. Acute abdomen in systemic lupus erythematosus: the importance of early laparotomy. Am J Med [Internet]. 1997; 103:100-5.

Molloy D, Molloy A, O’Loughlin C, Falconer M, Hennessy M. Inappropriate use of proton pump inhibitors. Ir J Med Sci. 2010; 179:73-5.

Musaev S, Novikova A, Shershevskaya A. Morphometric and immunohistochemical features of the gastric and duodenal mucosa in systemic lupus erythematosus. Bull Exp. 1991; 111:203-5.

Nardino RJ, Vender RJ, Herbert PN. Overuse of acidsuppressive therapy in hospitalized patients. Am J Gastroenterol. 2000; 95:3118-22.

Nasser SC, Nassif JG, Dimassi HI. Clinical and cost impact of intravenous proton pump inhibitor use in non-ICU patients. World $\mathbf{J}$ Gastroenterol. 2010; 16:982-6.

Naunton M, Peterson GM, Bleasel MD. Overuse of proton pump inhibitors. J Clin Pharm Ther. 2000; 25:333-40.

Niklasson A, Bajor A, Bergendal L, Simrén M, Strid H, Björnsson E. Overuse of acid suppressive therapy in hospitalised patients with pulmonary diseases. Respir Med. 2003; 97:1143-50.

Ntaios G, Chatzinikolaou A, Kaiafa G, Savopoulos C, Hatzitolios A, Karamitsos D. Evaluation of use of proton pump inhibitors in Greece. Eur J Intern Med. 2009; 20:171-3.

Oh AL, Tan AG, Phan HS, Lee BC, Jumaat N, Chew SP, et al. Indication of acid suppression therapy and predictors for the prophylactic use of proton-pump inhibitors vs. histamine-2 receptor antagonists in a Malaysian tertiary hospital. Pharm Pract . 2015; 13:633-633.

Pinto-Sanchez MI, Yuan Y, Bercik P, Moayyedi P. Proton pump inhibitors for functional dyspepsia. Cochrane Database of Systematic Reviews. 2014; 7.

Ramirez E, Lei SH, Borobia AM, Pinana E, Fudio S, Munoz R, et al. Overuse of PPIs in patients at admission, during treatment, and at discharge in a tertiary Spanish hospital. Curr Clin Pharmacol. 2010; 5:288-97.

Sandig C, Flechtenmacher C, Stremmel W, Eisenbach C. Pantoprazole induces severe acute hepatitis. Z Gastroenterol. 2011; 49:207-10.

Suzuki M, Suzuki H, Hibi T. Proton Pump Inhibitors and Gastritis. J Clin Biochem Nutr. 2008; 42:71-5.

Tleyjeh IM, Bin Abdulhak AA, Riaz M, Alasmari FA, Garbati MA, AlGhamdi M, et al. Association between Proton Pump Inhibitor Therapy and Clostridium difficile Infection: A Contemporary Systematic Review and Meta-Analysis. PLoS One. 2012; 7:1-12. 
Tze C, Chia W, Lim WP, Kien C, Vu F. Inappropriate use of proton pump inhibitors in a local setting. Singapore Med J. 2014; 55:3636.

Xu D, Yang H, Lai CC, Li P, Zhang X, Yang XO, Zhang FC, Qian JM. Clinical analysis of systemic lupus erythematosus with gastrointestinal manifestations. Lupus. 2010; 19:866-9.

Yu EW, Bauer SR, Bain PA, Bauer DC. Proton pump inhibitors and risk of fractures: A meta-analysis of 11 international studies. Am $\mathrm{J}$ Med. 2011; 124:519-26.

\section{How to cite this article:}

Elnaem MH, Mohamed MH, Nazar AH, Ibrahim RNK. Evaluation of Proton Pump Inhibitors Prescribing among Non-Critically Ill Hospitalized Patients in a Malaysian Tertiary Hospital. J App Pharm Sci, 2017; 7 (12): 077-083. 